\title{
A VERSION OF A THEOREM OF DAHLBERG FOR THE SUBELLIPTIC DIRICHLET PROBLEM
}

\author{
Luca Capogna, Nicola Garofalo, and Duy-Minh Nhieu
}

\section{Introduction}

In 1977 B. Dahlberg [7] proved his celebrated theorem stating that for a bounded Lipschitz domain in $\mathbb{R}^{n}$ harmonic and surface measure are mutually absolutely continuous and, furthermore, the Radon-Nikodym derivative of harmonic measure with respect to surface measure satisfies a reverse Hölder inequality. The aim of this note is to announce a similar theorem for sub-Laplacians and provide a complete solution for the Dirichlet problem for $L^{p}$ boundary data for a large class of domains. Such class, however, does not coincide with that in Dahlberg's theorem. This important aspect separates in an essential way our results from their predecessors for the Laplace equation and is connected with the existence of characteristic points on the boundary of the domain. This point will be clarified by the subsequent discussion. Our main results are Theorems $1.2,1.4,1.5$ and 1.6. The operators that we consider are sub-Laplacians $\mathcal{L}=\sum_{j=1}^{m} X_{j}^{*} X_{j}$, where $X=\left\{X_{1}, \ldots, X_{m}\right\}$ is a system of $C^{\infty}$ vector fields satisfying the finite rank condition on the Lie algebra

$$
\operatorname{rankLie}\left[X_{1}, \ldots, X_{m}\right](x)=n
$$

at every $x \in \mathbb{R}^{n}$. Denote by $d(x, y)$ the Carnot-Carathéodory distance associated to $X$ and let $B_{d}(x, r)=\left\{y \in \mathbb{R}^{n} \mid d(x, y)<r\right\}$. The fundamental properties of the metric balls were established by Nagel, Stein and Wainger in [22]. Given an open set $\Omega \subset \mathbb{R}^{n}$, a distributional solution of $\mathcal{L} u=0$ in $\Omega$ is called $\mathcal{L}$ harmonic. Hörmander's hypoellipticity theorem [14] guarantees that every $\mathcal{L}$ harmonic function is in $C^{\infty}(\Omega)$, hence it is a classical solution of $\mathcal{L} u=0$. When $\Omega$ is also bounded, the Dirichlet problem consists in finding a $\mathcal{L}$-harmonic function $u$ in $\Omega$ which takes some prescribed values $\phi$ on $\partial \Omega$. An important consequence of the pioneering work of Bony [1] is that for every $\phi \in C(\partial \Omega)$ there exists a unique $u=H_{\phi}^{\Omega}$ which solves the Dirichlet problem in a generalized sense. This allows to define the $\mathcal{L}$-harmonic measure $d \omega^{x}$ as the unique probability measure on $\partial \Omega$ such that

$$
H_{\phi}^{\Omega}(x)=\int_{\partial \Omega} \phi(Q) d \omega^{x}(Q)
$$

Received February 6, 1998.

Second author was supported in part by NSF Grant No. DMS-9706892 
The representation (1.1) continues to hold if $\phi \in L^{1}\left(\partial \Omega, d \omega^{x_{o}}\right)$, for some $x_{o} \in \Omega$. If $\Omega$ satisfies in addition the analogue of the uniform outer corkscrew condition introduced in [17], then by the results in [5], [9] the generalized solution $H_{\phi}^{\Omega}$ in (1.1) belongs to a Hölder class $\Gamma_{d}^{\alpha}(\bar{\Omega})$, if the boundary datum $\phi$ is in $\Gamma_{d}^{\alpha}(\partial \Omega)\left(\Gamma_{d}^{\alpha}\right.$ is the Hölder class with respect to $\left.d(x, y)\right)$. Suppose now that $\Omega$ be smooth and denote by $\Sigma=\left\{x \in \partial \Omega \mid X_{j}(x) \in T_{x}(\partial \Omega), j=1, \ldots, m\right\}$ the characteristic set of the system $X$. The classical results of Kohn and Nirenberg [19] guarantee that away from $\Sigma$ the solution to the Dirichlet problem, with $\phi \in$ $C^{\infty}(\bar{\Omega})$, is smooth up to the boundary. D. Jerison [15] first studied the Dirichlet problem at characteristic points for the sub-Laplacian in the Heisenberg group $\mathbb{H}^{n}$, and for the closely related Baouendi-Grushin operator. The results in [15], [16] show that regardless of the smoothness of the domain and of the boundary data one cannot expect in general more than $\Gamma_{d}^{\alpha}$ regularity in the neighborhood of a characteristic point. This is a new phenomenon which is reminiscent of boundary value problems for elliptic operators in non-smooth domains. Since the smoothness of the ground domain does not suffice to guarantee higher regularity of the solution up to the boundary, one has to turn the attention to geometric properties. In this respect a remarkable negative phenomenon is the lack of Lipschitz domains. A striking example due to D. Jerison shows that such class is practically empty, so one must abandon the idea of a Lipschitz domain too, see [4] for a detailed discussion.

We thus come to one of the central question for the results in this note: What are the domains that in the Dirichlet problem for sub-Laplacians replace the class of Lipschitz domains? The answer is contained in the following

Definition 1.1. Given a system $X=\left\{X_{1}, \ldots, X_{m}\right\}$ we say that a smooth, connected, bounded open set $\Omega \subset \mathbb{R}^{n}$ is admissible for the Dirichlet problem ( $\left.A D P_{X}\right)$ for the sub-Laplacian associated to $X$, if $\Omega$ satisfies the uniform outer $\mathcal{L}$-ball condition and is X-NTA.

A bounded open set $\Omega \subset \mathbb{R}^{n}$ is said to satisfy the uniform outer $\mathcal{L}$-ball condition if one can find $R_{o}>0$ such that for every $Q \in \partial \Omega$ and for every $0<r<R_{o}$ there exists a $\mathcal{L}$-ball $B\left(Q_{o}, r\right)$ for which

$$
Q \in \partial B\left(Q_{o}, r\right), \quad B\left(Q_{o}, r\right) \cap \Omega=\varnothing
$$

holds. Here, the sets $B(x, r)$ are the interior of suitably rescaled level sets of the positive fundamental solution $\Gamma(x, y)$ of the sub-Laplacian, see [6], [3]. We note that for groups of Heisenberg type [18] the $\mathcal{L}$-balls coincide with the balls in the nonisotropic gauge. The class of $X$-NTA domains is a generalization of that introduced in [17].

The question of producing examples of $A D P_{X}$ domains has basic relevance, of course. It turns out that this task requires a very delicate analysis and considerable work. We remark that every $C^{1,1}$ domain is $A D P_{X}$ with respect to the system $X=\left\{\frac{\partial}{\partial x_{1}}, \ldots, \frac{\partial}{\partial x_{n}}\right\}$, whose Carnot-Carathéodory metric is the standard Euclidean distance $d(x, y)=|x-y|$. In this context, another important class 
of examples is provided by convex domains. They clearly satisfy the outer ball condition with respect to $|\cdot|$ and moreover every convex domain is Lipschitz, hence NTA according to [17]. It is interesting that the notion of Euclidean convexity (at the level of the Lie algebra) also plays a role in constructing examples of $A D P_{X}$ domains in the subelliptic setting. For instance we prove that in any group of Heisenberg type $\boldsymbol{G}$ the gauge balls are $A D P_{X}$ domains (these sets are convex at the level of the Lie algebra). As a consequence of this result and of our general Theorems 1.3, 1.4, 1.5 and 1.6 we obtain the following

Theorem 1.2. Let $\boldsymbol{G}$ be a group of Heisenberg type and denote by $B$ the gauge unit ball centered at the group identity e. Let $P(Q)=P(e, Q)$ be the Poisson kernel (relative to $B$ and to $\mathcal{L}$ ) defined in (1.6). For every $p>1$ there exist constants $C=C(\boldsymbol{G}, p)>0, R_{o}=R_{o}(\boldsymbol{G})>0$ such that for every $Q_{o} \in \partial B$ and $0<r<R_{o}$ one has

$$
\left(\frac{1}{\sigma\left(\Delta\left(Q_{o}, r\right)\right)} \int_{\Delta\left(Q_{o}, r\right)} P(Q)^{p} d \sigma\right)^{\frac{1}{p}} \leq C \frac{1}{\sigma\left(\Delta\left(Q_{o}, r\right)\right)} \int_{\Delta\left(Q_{o}, r\right)} P(Q) d \sigma .
$$

As a consequence of (1.3) and (1.9), $\mathcal{L}$-harmonic measure $d \omega^{e}$ and surface measure $d \sigma$ are mutually absolutely continuous. Moreover, $L^{p}(\partial \Omega, d \sigma) \subset L^{1}(\partial \Omega$, $\left.d \omega^{e}\right)$. Finally, for every $\phi \in L^{p}(\partial \Omega, d \sigma)$ one has with $H_{\phi}^{\Omega}$ as in (1.1)

$$
\left\|N_{\alpha}\left(H_{\phi}^{\Omega}\right)\right\|_{L^{p}(\partial \Omega, d \sigma)} \leq C\|\phi\|_{L^{p}(\partial \Omega, d \sigma)},
$$

and $H_{\phi}^{\Omega}(Q)$ converges nontangentially to $\phi(Q)$ for a.e. $Q \in \partial \Omega$ with respect to $d \sigma$.

Here, $N_{\alpha}\left(H_{\phi}^{\Omega}\right)$ represents the nontangential maximal function of $H_{\phi}^{\Omega}$ defined in (1.14) below. We emphasize that, due to the presence of characteristic points, surface measure $d \sigma$ can be quite singular. The fact that we can solve the Dirichlet problem with respect to $d \sigma$ represents a sharp aspect. Theorem 1.2 provides a complete solution to the Dirichlet problem for the gauge balls in groups of Heisenberg type. Such groups were introduced by Kaplan [18] as a direct generalization of the Heisenberg group $\mathbb{H}^{n}$ in connection with hypoellipticity questions. Groups of Heisenberg type are important since they include the nilpotent component in the Iwasawa decomposition of simple groups of rank one. Before we continue with the description of the other results, we pause to return to D. Jerison's negative example for higher boundary regularity in the Heisenberg group $\mathbb{H}^{n}[15]$. The latter is given by a domain whose complement near the characteristic point $e=(0,0,0)$ is the region inside the paraboloid $M=\left\{(x, y, t) \in \mathbb{H}^{n} \mid t=-C\left(|x|^{2}+|y|^{2}\right)\right\}$, with $C>0$. A simple calculation shows that such domain fails to fulfill the outer $\mathcal{L}$-ball condition in (1.2). This should not be surprising since with the glasses of the sub-Riemannian geometry of $\mathbb{H}^{n}$ a paraboloid looks like a standard Euclidean cone. In fact, one should think of Jerison's construction as the analogue for the Heisenberg group of the 
classical counterexamples to the boundary boundedness of the gradient of the Green function for nonconvex Euclidean cones.

Concerning the uniform outer $\mathcal{L}$-ball condition in Definition 1.1 we mention that it is inspired to a beautiful idea in a classical paper of Poincaré [23]. In 1991 one of us, N. G., conjectured that such condition should imply higher regularity of the solution at characteristic points. We prove that this conjecture is true: Let $\Omega \subset \mathbb{R}^{n}$ be a $C^{\infty}$ connected open set satisfying the uniform outer $\mathcal{L}$-ball condition. There exists a constant $C=C(X, \Omega)>0$ such that for each $x, y \in \Omega$ with $x \neq y$ one has

$$
\begin{aligned}
& \text { (i) } \quad G(x, y) \leq C d(y, \partial \Omega) \frac{d(x, y)}{\left|B_{d}(x, d(x, y))\right|} \\
& \text { (ii) }|X G(x, y)| \leq C \frac{d(x, y)}{\left|B_{d}(x, d(x, y))\right|}
\end{aligned}
$$

We remark explicitly that (ii) implies in particular that if for a fixed $x_{o} \in \Omega$ we let $g(y)=G\left(x_{o}, y\right)$, then the horizontal gradient $X g=\left(X_{1} g, \ldots, X_{m} g\right)$ is in $L^{\infty}$ in a sufficiently small neighborhood of $\partial \Omega$. Since we are interested in local questions we can without loss of generality suppose that $\Omega=\left\{x \in \mathbb{R}^{n} \mid \rho(x)<0\right\}$ for some $\rho \in C^{\infty}\left(\mathbb{R}^{n}\right)$ satisfying $|D \rho(x)| \geq \alpha_{\Omega}^{-1}>0$, for every $x \in K$, for some relatively compact neighborhood $K$ of $\partial \Omega$. The outward pointing unit normal to $\partial \Omega$ is $\eta=\frac{D \rho}{|D \rho|}$. We let $w(x)=|X \rho(x)|$, and notice that $w(x)=0$ for every $x \in \Sigma$, whereas $w(x)>0$ for $x \in \partial \Omega \backslash \Sigma$. Surface measure on $\partial \Omega$ will be indicated by $\sigma=H^{n-1}\left\lfloor\partial \Omega\right.$ (here, $H^{n-1}$ denotes the usual $(n-1)$-dimensional Hausdorff measure). We define a new measure on $\partial \Omega$ by letting

$$
d \mu=w d \sigma
$$

By [19] one has for the Green function $G(x, y)$ for $\mathcal{L}$ and $\Omega$ : For every fixed $x \in \Omega$ and $Q_{o} \in \partial \Omega \backslash \Sigma$, there exists a sufficiently small neighborhood $V$ of $Q_{o}$, such that $y \rightarrow G(x, y)$ is $C^{\infty}$ in $V \cap \bar{\Omega}$. This being said, we define two functions $P$ and $K$ on $\Omega \times(\partial \Omega \backslash \Sigma)$

$$
\begin{aligned}
& P(x, Q)=\left[-\sum_{j=1}^{m} X_{j} G(x, \cdot)<X_{j}, \eta>\right](Q), \\
& K(x, Q)=w(Q)^{-1} P(x, Q) .
\end{aligned}
$$

For every $x \in \Omega$ we extend with zero the definition of $P(x, \cdot)$ and $K(x, \cdot)$ to all of $\partial \Omega$. Thanks to a result of Franchi and Wheeden [13] one has $\sigma(\Sigma)=0$, so that the extended functions coincide $\sigma$-a.e. with the original ones. The estimates lead to the following basic representation formula: Let $\Omega$ be a $C^{\infty}$ connected, 
open set possessing the uniform outer $\mathcal{L}$-ball condition. For every $\phi \in C(\partial \Omega)$ one has

$$
H_{\phi}^{\Omega}(x)=\int_{\partial \Omega} \phi(Q) K(x, Q) d \mu=\int_{\partial \Omega} \phi(Q) P(x, Q) d \sigma, \quad x \in \Omega .
$$

In particular, the $\mathcal{L}$-harmonic measure $d \omega^{x}$ is absolutely continuous with respect to $d \mu$ and with respect to $d \sigma$. Moreover

$$
\frac{d \omega^{x}}{d \mu}(Q)=K(x, Q), \quad \frac{d \omega^{x}}{d \sigma}(Q)=P(x, Q) .
$$

We mention that a similar representation formula has been recently independently obtained in an interesting paper by Lanconelli and Uguzzoni [20], but only for the special setting of the Heisenberg group. An immediate consequence of (1.4), (1.7) is the following a priori estimates in $L^{p}$ in the Dirichlet problem when the boundary datum is a continuous function. Let $\phi \in C(\partial \Omega)$, and denote by $H_{\phi}^{\Omega}$ the Perron-Wiener-Brelot solution to the Dirichlet problem in (1.1): For each $1 \leq p<\infty$ there exists $C=C(p, X, \Omega)$ such that

$$
\left\|H_{\phi}^{\Omega}\right\|_{L^{p}(\Omega)} \leq C\|\phi\|_{L^{p}(\partial \Omega)} .
$$

Solvability with data in Lebesgue classes on the boundary of $\Omega$ requires, however, a much deeper analysis. The first observation is that the outer $\mathcal{L}$-ball condition alone does not guarantee the development of a rich potential theory. For instance, it may not be possible to find good nontangential regions of approach to the boundary from within the domain. This is the point at which the notion of nontangentially accessible (NTA) domain comes into play. Being purely metrical it is better suited, than that of a Lipschitz (or smooth) domain, to the study of boundary value problems for sub-Laplacians. The drawback is that most of the central results in the theory become much harder to prove in this setting, since the geometry is considerably more complicated than the Euclidean one, or else they are plainly false. Furthermore, the important task of constructing examples offers serious difficulties due to the presence of characteristic points on the boundary of the domain. These obstacles were overcome in [4], where two of us studied the boundary behavior of $\mathcal{L}$-harmonic functions in $X$-NTA domains, i.e., domains which are NTA with respect to the CarnotCarathéodory metric associated to a system $X$. Two central results from [4] which play a fundamental role in the proof of Theorems 1.2, 1.4 and 1.5 are the doubling condition for $\mathcal{L}$-harmonic measure and the comparison theorem. The former states that, given a $X$-NTA domain $\Omega$, there exist $C>0, R_{o}>0$ and $a>1$ such that for any $Q \in \partial \Omega$ and $0<r<R_{o}$ one has

$$
\omega^{x}(\Delta(Q, 2 r)) \leq C \omega^{x}(\Delta(Q, r))
$$

for any $x \in \Omega \backslash B_{d}(Q, a r)$. Here, we have let $\Delta(Q, r)=B_{d}(Q, r) \cap \partial \Omega$. The second result from [4] that we need is a comparison theorem which roughly speaking 
states that if two nonnegative $\mathcal{L}$-harmonic functions vanish continuously on a surface ball $\Delta(Q, r)$ of a given $X$-NTA domain, they do so at an equivalent rate.

We now return to the measure $d \mu$ defined in (1.4). A key fact (established in [10]) is that $d \mu$ is equivalent to the notion of relative $X$-perimeter introduced in [2]. This fact essentially says that in a Carnot group with homogeneous dimension $Q$ (see [12]), the measure $d \mu$ is like the restriction to $\partial \Omega$ of the $(Q-$ 1)-dimensional Hausdorff measure constructed with the Carnot-Carathéodory distance. Thereby, $d \mu$ should behave much better than $d \sigma$. This observation is justified by the following theorem which plays an important role in our results.

Theorem 1.3. Let $\boldsymbol{G}$ be a Carnot group of arbitrary step with homogeneous dimension $Q$, and consider a smooth domain $\Omega=\{p \in \boldsymbol{G} \mid \rho(p)<0\}$, where $\rho \in C^{\infty}(\boldsymbol{G})$ is a defining function for $\Omega$. There exist $C, R_{o}>0$ depending on $\boldsymbol{G}$ and $\Omega$ such that for every $Q_{o} \in \partial \Omega$ and $0<r \leq R_{o}$ one has

(i) $\left(\sup _{Q \in \Delta\left(Q_{o}, r\right)}|X \rho|(Q)\right) \sigma\left(\Delta\left(Q_{o}, r\right)\right) \leq C r^{Q-1}$.

(ii) $\mu\left(\Delta\left(Q_{o}, r\right)\right) \leq C r^{Q-1}$.

In the statement of Theorem 1.3 we have, with abuse of notation, continued to denote by $d \sigma$ the composition of surface measure on the Lie algebra of $\boldsymbol{G}$ with the exponential map based at $Q_{o}$. Although we cannot go into a detailed discussion of (i) and (ii), it should be clear that (ii) expresses the "good scaling properties" of $d \mu$. On the other hand, (i) says that at the characteristic points $d \sigma$ can be quite singular. Theorem 1.3 extends a result that in the special case of the Heisenberg group $\mathbb{H}^{n}$ was established by C. Romero [24], and subsequently and independently by M. Mekias [21], in their respective Ph. D. Dissertations.

We are now ready to state our main results.

Theorem 1.4. Let $\Omega \subset \mathbb{R}^{n}$ be a ADP $P_{X}$ domain. Suppose that there exist $M, R_{o}>0$ such that for every $Q \in \partial \Omega$ and $0<r<R_{o}$

$$
\mu(\Delta(Q, r)) \leq M \frac{\left|B_{d}(Q, r)\right|}{r} .
$$

For every $p>1$ and any fixed $x_{o} \in \Omega$ one can find positive constants $C, R_{1}$, depending on $p, M, R_{o}, x_{o}$, and on the $A D P_{X}$ parameters, such that for $Q_{o} \in \partial \Omega$ and $0<r<R_{1}$ one has

$$
\begin{aligned}
& \left(\frac{1}{\mu\left(\Delta\left(Q_{o}, r\right)\right)} \int_{\Delta\left(Q_{o}, r\right)} K\left(x_{o}, Q\right)^{p} d \mu\right)^{\frac{1}{p}} \\
& \leq C \frac{1}{\mu\left(\Delta\left(Q_{o}, r\right)\right)} \int_{\Delta\left(Q_{o}, r\right)} K\left(x_{o}, Q\right) d \mu .
\end{aligned}
$$

As a consequence of (1.8),(1.10) and (1.11) we infer that $d \mu$ is also doubling. Furthermore, the measures $d \omega^{x}$ and $d \mu$ are mutually absolutely continuous. 
If instead of considering the measure $d \mu$ we work with surface measure $d \sigma$, then by imposing a stronger restriction on the nature of characteristic points we can prove a reverse Hölder inequality for the Poisson kernel $P(x, Q)$ defined in (1.6).

Theorem 1.5. Let $\Omega \subset \mathbb{R}^{n}$ be a $A D P_{X}$ domain. Suppose there exist $M, R_{o}>0$ such that for every $Q_{o} \in \partial \Omega$ and $0<r<R_{o}$

$$
\left(\max _{Q \in \Delta\left(Q_{o}, r\right)} w(Q)\right) \sigma\left(\Delta\left(Q_{o}, r\right)\right) \leq M \frac{\left|B\left(Q_{o}, r\right)\right|}{r} .
$$

We fix $x_{o} \in \Omega$. For every $p>1$ there exist positive constants $C, R_{1}$, depending on $p, M, R_{o}, x_{o}$, and on the $A D P_{X}$ parameters, such that for every $Q_{o} \in \partial \Omega$ and $0<r<R_{1}$ one has

$$
\begin{aligned}
& \left(\frac{1}{\sigma\left(\Delta\left(Q_{o}, r\right)\right)} \int_{\Delta\left(Q_{o}, r\right)} P\left(x_{o}, Q\right)^{p} d \sigma\right)^{\frac{1}{p}} \\
& \leq C \frac{1}{\sigma\left(\Delta\left(Q_{o}, r\right)\right)} \int_{\Delta\left(Q_{o}, r\right)} P\left(x_{o}, Q\right) d \sigma .
\end{aligned}
$$

Moreover, thanks to (1.8),(1.12) and (1.13), the measure $d \sigma$ is doubling, and $d \omega^{x}, d \mu$ and $d \sigma$ are mutually absolutely continuous.

Theorems 1.4 and 1.5 allow to solve the Dirichlet problem for boundary data in $L^{p}$. This consequence is not straightforward, however, but as in the classical case rests on the development of a Fatou theory. This was done in [4] and the results that we describe below rely in an essential way on a combination of the Fatou theory and of Theorems 1.4, 1.5. We need to introduce a definition. For any $Q \in \partial \Omega$ and $\alpha>0$ a nontangential region at $Q$ is given by

$$
\Gamma_{\alpha}(Q)=\{x \in \Omega \mid d(x, Q) \leq(1+\alpha) d(x, \partial \Omega)\} .
$$

Given a function $u \in C(\Omega)$, the $\alpha$-nontangential maximal function of $u$ at $Q$ is defined by

$$
N_{\alpha}(u)(Q)=\sup _{x \in \Gamma_{\alpha}(Q)}|u(x)| .
$$

Under the assumptions of Theorems 1.4, 1.5 one obtains the important conclusion that the spaces $L^{p}(\partial \Omega, d \mu), L^{p}(\partial \Omega, d \sigma)$ are continuously embedded in $L^{1}\left(\partial \Omega, d \omega^{x_{o}}\right), x_{o} \in \Omega$. Therefore, if $\phi$ is in either of these spaces we can write $H_{\phi}^{\Omega}$ as in (1.1). From the results in [4] we know that $H_{\phi}^{\Omega}$ converges non-tangentially to $\phi$ for a.e. point $Q \in \partial \Omega$ with respect to $d \omega^{x_{o}}$. Combining these facts with Theorems 1.4, 1.5, we obtain the solvability of the Dirichlet problem for $A D P_{X}$ domains when the boundary datum is in $L^{p}$ of the boundary, for $1<p<\infty$, with respect to the measures $d \mu$ or $d \sigma$ respectively.

Theorem 1.6. Let $\Omega \subset \mathbb{R}^{n}$ be a $A D P_{X}$ domain. 
(i) Suppose that $d \mu$ satisfy (1.10). For every $p>1$ there exists a constant $C>0$ depending on $p$ and on $\Omega$ such that if $\phi \in L^{p}(\partial \Omega, d \mu)$, then

$$
\left\|N_{\alpha}\left(H_{\phi}^{\Omega}\right)\right\|_{L^{p}(\partial \Omega, d \mu)} \leq C\|\phi\|_{L^{p}(\partial \Omega, d \mu)} .
$$

Furthermore, $H_{\phi}^{\Omega}(Q)$ converges nontangentially to $\phi(Q)$ for a.e. $Q \in \partial \Omega$ with respect to $d \mu$.

(ii) Assume that do satisfy (1.12). For every $p>1$ one can find a $C>0$ depending on $p$ and on $\Omega$ for which one has for $\phi \in L^{p}(\partial \Omega, d \sigma)$

$$
\left\|N_{\alpha}\left(H_{\phi}^{\Omega}\right)\right\|_{L^{p}(\partial \Omega, d \sigma)} \leq C\|\phi\|_{L^{p}(\partial \Omega, d \sigma)} .
$$

Moreover, $H_{\phi}^{\Omega}(Q)$ converges nontangentially to $\phi(Q)$ for a.e. $Q \in \partial \Omega$ with respect to $d \sigma$.

Theorems 1.4 and 1.5 constitute the analogue in the subelliptic Dirichlet problem of Dahlberg's theorem cited in the opening. In the classical setting the solvability of the Dirichlet problem in the range $1<p<\infty$ was obtained by Dahlberg [8] for $C^{1}$ domains using his results in [7], and also independently by Fabes, Jodeit and Rivière [11] by the method of layer potentials. As witnessed by Theorem 1.3 the hypothesis (1.10), (1.12) are natural requirements. They are fulfilled by any smooth domain in every Carnot group.

\section{Acknowledgements}

We thank David Jerison for stimulating conversations through the years. We also thank Qing Han and Mei-Chi Shaw for pointing to our attention the relevance of Euclidean convexity in the subelliptic Dirichlet problem.

\section{References}

1. J. M. Bony, Principe du maximum, inégalité de Harnack et unicité du problème de Cauchy pour les operateurs elliptique degeneres, Ann. Inst. Fourier, Grenoble (1) 119 (1969), 277304.

2. L. Capogna, D. Danielli, and N. Garofalo, An isoperimetric inequality and the geometric Sobolev embedding for vector fields, Comm. Anal. Geom. (2) 2 (1994), 203-215.

3. __ Capacitary estimates and the local behavior of solutions of nonlinear subelliptic equations, Amer. J. Math. 118 (1997), 1153-1196.

4. L. Capogna and N. Garofalo, Boundary behavior of nonnegative solutions of subelliptic equations in NTA domains for Carnot-Carathéodory metrics, J. Fourier Analysis and Appl., to appear.

5. G. Citti, Wiener estimates at boundary points for Hörmander's operators, Boll. Un. Mat. Ital. B (7) 2 (1988), 667-681.

6. G. Citti, N. Garofalo, and E. Lanconelli, Harnack's inequality for sum of squares of vector fields plus a potential, Amer. J. Math. 115 (1993), 699-734.

7. B. E. J. Dahlberg, Estimates of harmonic measure, Arch. Rat. Mech. An. 65 (1977), $272-288$.

8. 13-24.

9. D. Danielli, Regularity at the boundary for solutions of nonlinear subelliptic equations, Indiana J. Math. 44 (1995), 269-286. 
10. D. Danielli, N. Garofalo, and D. M. Nhieu, Trace inequalities for Carnot-Carathéodory spaces and applications, Annali Sc. Normale Sup. Pisa, to appear.

11. E. B. Fabes, M. Jodeit, Jr., and N. Rivière, Potential techniques for boundary value problems on $C^{1}$-domains, Acta Math. 141 (1978), 165-186.

12. G. Folland, Subelliptic estimates and function spaces on nilpotent Lie groups, Ark. Math. 13 (1975), 161-207.

13. B. Franchi and R. L. Wheeden, Compensation couples and isoperimetric estimates for vector fields, Coll. Math. 74 (1997), 9-27.

14. H. Hörmander, Hypoelliptic second-order differential equations, Acta Math. 119 (1967), 147-171.

15. D. Jerison, The Dirichlet problem for the Kohn Laplacian on the Heisenberg group, Parts I and II, J. Funct. Analysis 43 (1981), 97-142.

16. _ Boundary regularity in the Dirichlet problem for $\square_{b}$ on CR manifolds, Comm. Pure Appl. Math. 36 (1983), 143-181.

17. D. Jerison and C. E. Kenig, Boundary behavior of harmonic functions in non-tangentially accessible domains, Adv. Math. 46 (1980), 80-147.

18. A. Kaplan, Fundamental solutions for a class of hypoelliptic PDE generated by composition of quadratic forms, Trans. Amer. Math. Soc. 258 (1980), 147-153.

19. J. J. Kohn and L. Nirenberg, Non-coercive boundary value problems, Comm. Pure and Appl. Math. 18 (1965), 443-492.

20. E. Lanconelli and F. Uguzzoni, On the Poisson kernel for the Kohn Laplacian, Rend. Mat. Appl. 17 (1997), 659-677.

21. M. Mekias, Restriction to hypersurfaces of non-isotropic Sobolev spaces, M.I.T. Ph.D Thesis, (1993).

22. A. Nagel, E. M. Stein and S. Wainger, Balls and metrics defined by vector fields I: basic properties, Acta Math. 155 (1985), 103-147.

23. H. Poincaré, Sur les equations aux dérivées partielles de la physique mathématique, Amer. J. Math. 12 (1890), 211-294.

24. C. Romero, Potential theory for the Kohn Laplacian on the Heisenberg group, Univ. of Minnesota Ph. D. Thesis (1991).

Courant Institute, 251 Mercer Street, New York, NY 10012

E-mail address: capogna@math1.cims.nyu.edu

Department of Mathematics, Purdue University, West Lafayette IN 47907-1935

E-mail address: garofalo@math.purdue.edu

Institute of Mathematics, Academia Sinica, Nankang, Taipei 11529, Taiman R. O. C. E-mail address: dmnhieu@math.sinica.edu.tw 